\title{
Implementing Triage Standing Orders in the Emergency Department Leads to Reduced Physician-to-Disposition Times
}

\author{
Charles W. Hwang, Thomas Payton, Emily Weeks, and Michelle Plourde \\ Department of Emergency Medicine, University of Florida College of Medicine, 1329 SW 16th Street, P.O. Box 100186, Gainesville, \\ FL 32610-0186, USA \\ Correspondence should be addressed to Thomas Payton; tfpayton@ufl.edu
}

Received 13 January 2016; Accepted 31 May 2016

Academic Editor: Patrick Schober

Copyright (C) 2016 Charles W. Hwang et al. This is an open access article distributed under the Creative Commons Attribution License, which permits unrestricted use, distribution, and reproduction in any medium, provided the original work is properly cited.

\begin{abstract}
Emergency departments (EDs) throughout USA have improvised various processes to curb the "national epidemic" termed ED "crowding." Standing orders (SOs), one such process, are medical orders approved by the medical director and entered by nurses when patients cannot be seen expeditiously, expediting medical decision-making and decreasing length of stay (LOS) and time to disposition. This retrospective cohort study evaluates the impact of SOs on ED LOS and disposition time at a large university ED. Results indicate that SOs significantly improve ED throughput by reducing disposition time by up to $16.9 \%(p=0.04)$, which is especially significant in busy ED settings. SOs by themselves are not sufficient for a complete diagnostic assessment. Strategies such as having a provider in the waiting area may help make key decisions earlier.
\end{abstract}

\section{Introduction}

Background. Throughout the United States, emergency departments (EDs) are called upon to care for more and more patients; from 1995 to 2005, annual ED visits increased by $20 \%$ and ED utilization increased by $7 \%$, from 36.9 to 39.6 ED visits per 100 persons [1-5]. In addition, the multifaceted scope of emergency care has broadened to encompass care of indigent patients, stabilization of the acutely ill, proficiency in procedures, medical observation, and primary healthcare [4]. Despite the increase in patient volume and complexity, hospital and ED resources have not increased proportionately. Instead, during this same period, the number of EDs decreased by 381 , the number of hospitals decreased by 535 , and the number of inpatient hospital beds decreased by 134,000 [1-4].

These trends have resulted in ED "crowding" described by the American College of Emergency Physicians (ACEP) as "when the identified need for emergency services exceeds available resources for patient care in the $\mathrm{ED}$, hospital, or both." [6]. As the ED system is overburdened by crowding, the quality and safety of emergency care decline.
Importance. In response to $\mathrm{ED}$ crowding, multiple $\mathrm{ED}$ processes have been implemented across the country to target input, throughput, and output factors to increase ED efficiency; standing triage orders are one such process $[5,7,8]$. Standing triage orders are orders derived from consensus and clinical practice guidelines for specific patient complaints or conditions; they have been implemented in EDs as a strategy to improve ED efficiency. In theory, standing orders are implemented by nursing staff prior to physician evaluation, initiating the diagnostic evaluation earlier, expediting medical decision-making, improving ED throughput, increasing efficiency, and improving patient care and outcomes.

Goal of This Study. Standing orders were developed at our institution for discrete patient complaints or conditions and are initiated by nursing staff if patients cannot be placed in a care area within 15 minutes of arrival or if a provider cannot see patients within 15 minutes of being roomed in a care area. Chest pain is the most common complaint for patients that present to the ED.

The chest pain standing order set was developed for patients who present with chest pain, chest pressure, dyspnea, 
arm or shoulder pain or paresthesia, syncope, near-syncope, and palpitations. The components of the order set are listed as follows.

Chest Pain Order Set. Standing orders are developed based on consensus guidelines, approved by the medical director, and implemented by the nursing staff if patients are not able to be seen in a timely fashion by provider staff.

\author{
Chest pain tests include the following: \\ Complete blood count. \\ Basic metabolic panel. \\ Troponin T. \\ Total creatine kinase. \\ Creatine kinase-MB. \\ Prothrombin time/international normalized \\ ratio. \\ Electrocardiogram. \\ Chest X-ray. \\ Digoxin (if in medication list). \\ Aspirin $324 \mathrm{mg}$ orally.
}

The purpose of this study was to analyze the effects and limitations of standing triage orders for patients presenting with chest pain. Primary outcomes included ED length of stay (LOS) and the time from provider evaluation to patient disposition, both surrogate markers for ED throughput. We hypothesized that the implementation of standing orders would shorten ED LOS and time to disposition.

\section{Materials and Methods}

2.1. Study Design. We conducted a retrospective cohort study of patients treated at a hospital-based ED during a 5-month period beginning in March 2014 and ending in July 2014. The ED is a large academic university-based 66-bed ED, a designated Level I trauma center, with an annual volume of 67,172 adult visits in 2014. Standing orders were implemented at the hospital ED starting in April 2014. All adult patients who presented between April 1, 2014, and July 31, 2014 (4month duration), with chest pain and who could not be placed in a care area within 15 minutes of arrival or could not be seen by a provider within 15 minutes of being roomed were eligible for standing orders. For waiting room patients unable to be roomed within the $\mathrm{ED}$, the waiting room triage nurse initiates the standing orders. For patients that are roomed but unable to be evaluated in an expeditious manner, the patient's nurse initiates the standing orders. Those patients who had chest pain-specific standing orders initiated were eligible for enrollment in the "standing orders" cohort in this study. Patients who had standing orders unexpectedly cancelled (either by provider mistake or lab error) were not included in analysis.

To eliminate Hawthorne, effect and selection bias, the "no-standing orders" cohort included only patients who presented prior to the implementation of standing orders in April 2014. All adult patients who presented to the ED between
March 1, 2014, and March 13, 2014, with chest pain were eligible for enrollment in the "no-standing orders" cohort. Patients with chest pain who were transferred from outside facilities to our hospital-based ED for further evaluation and management were excluded from the study to eliminate bias between separate emergency departments.

The patient characteristics of both no-standing orders and standing orders cohorts were compared to assess similarity of patient populations. Primary outcomes included ED LOS and time from provider evaluation to patient disposition. Secondary outcomes included the number of additional labs or imaging studies that were ordered upon provider evaluation.

IRB approval (\#201400729) was obtained prior to the initiation of this study.

2.2. Data Collection. All patient data was extracted from the hospital's electronic medical record system, EPIC (Madison, WI), an integrated record of inpatient and outpatient patient care records, including physician and nursing notes, vital signs, laboratory values, imaging, and ED timeline and patient care activities. For each patient, collected data included (1) patient demographics (age, gender); (2) patient characteristics (chief complaint, vital signs); (3) clinical events (time of arrival, time of triage, time of orders being placed, lab result time, time of patient being roomed, and time of provider evaluation); (4) final disposition; (5) time of disposition; (6) exit time; and (7) number of additional provider orders (labs, imaging).

The electronic medical record system automatically records any change in the patient's location (waiting room, ED bed, hospital bed, and discharged) or status (admission, discharge, leaving against medical advice, leaving without being seen, and leaving during treatment). All other aforementioned data points were automatically populated in EPIC during the ED visit. All data was extracted from EPIC retrospectively. Extracted data was used to determine whether the standing order set partially or completely resulted prior to provider evaluation.

2.3. Data Analysis. Patient characteristics of those with and without standing orders were compared to ensure similarity between the two cohorts. Our primary outcome, time from provider evaluation to disposition, was compared among those with no-standing orders to all patients with standing orders. The "standing order" participants were further subdivided into two categories: (1) patients who had standing orders initiated and all labs resulted prior to provider evaluation and (2) patients who had standing orders initiated and some labs resulted prior to provider evaluation. Subgroup analysis was performed on these groups, in addition to overall data, to determine the effects of standing orders on patient care timelines.

Patient demographics were characterized using univariate analysis. Patient characteristics were then compared between the standing orders and no-standing orders cohorts using chi-square and student's $t$-test for categorical and quantitative variables, respectively. Primary ED LOS and duration between provider evaluation and disposition outcomes were 
TABLE 1: Characteristics of patients presenting with chest pain separated into standing orders and no-standing orders cohorts.

\begin{tabular}{lccc}
\hline & $\begin{array}{c}\text { Standing } \\
\text { orders } \\
(n=149)\end{array}$ & $\begin{array}{c}\text { No-standing } \\
\text { orders } \\
(n=152)\end{array}$ & $p$ value \\
\hline Male \% $(n)$ & $41.6 \%(62)$ & $49.3 \%(75)$ & 0.1781 \\
Admit \% $(n)$ & $55.7 \%(83)$ & $59.9 \%(91)$ & 0.4489 \\
Discharge \% $(n)$ & $37.6 \%(56)$ & $36.2 \%(55)$ & 0.4489 \\
Age (mean) & 46.9 & 47.8 & 0.6440 \\
\hline
\end{tabular}

assessed using student's $t$-test. Secondary outcomes were reported as means and were only applicable to the standing orders cohorts.

\section{Results}

The EMR team identified 155 patients who presented to the ED between April 1, 2014, and July 31, 2014, with chest pain and had standing orders initiated. Six patients had standing orders which were inadvertently cancelled, resulting in 149 patients in the "standing orders" cohort available for statistical analysis.

The EMR team also identified 161 patients who presented to the ED with chest pain prior to the implementation of standing orders, to serve as the "no-standing orders" control group. Nine of these patients were transferred from our freestanding ED and were thus censored from analysis, resulting in 152 patients with chest pain in the "no-standing orders" cohort.

Three hundred and one patients with chest pain were analyzed, with $49.5 \%$ (149) in the standing orders group and 50.5\% (152) in the no-standing orders group. Overall, $54.5 \%$ of patients with chest pain were female, and the overall mean age was 47.3. Table 1 displays the characteristics of patients with chest pain, separated by standing order versus no-standing order cohorts. For patients with chest pain, there was a reduction in the median "provider evaluation to disposition" time, from $154 \mathrm{~min}$ in the no-standing orders group to $128 \mathrm{~min}$ ( $26 \mathrm{~min}$ decrease, $16.9 \%, p=0.04$ ) if all diagnostic studies were completed before the patient was evaluated by a provider. ED LOS was higher among the standing orders group, at $614 \mathrm{~min}$, compared to $402 \mathrm{~min}$ in the no-standing orders group.

The overall success rate in getting lab and basic radiology orders completed before provider evaluation was 94 out of 149 (63.1\%). Among all patients in the standing orders group, $74.5 \%$ (111) of patients had additional labs ordered, with a mean of 3.4 additional labs, and $8.1 \%$ (12) of patients had additional radiology studies ordered, with a mean of 1.3 additional studies, and $32.2 \%$ (48) of patients had additional advanced radiology studies ordered, with a mean of 1.3 additional studies. The full data is reflected in Table 2.

\section{Discussion}

The Institute of Medicine (IOM) Committee on the Future of Emergency Care in the United States Health System has referred to ED crowding as "a national epidemic" $[2,3,9]$. As the ED system is overburdened by crowding, the quality and safety of emergency care decline. Direct consequences of crowding include increased wait times [4, 5, 10, 11], increased rates of patients leaving without being seen $[7,9-$ $11]$, prolonged LOS $[3,7,8]$, higher patient dissatisfaction $[3,5,7-9,11,12]$, poorer clinical outcomes $[3,5,7,9]$, delay in time-sensitive interventions $[4,7,10,11,13-15]$, increased adverse events [7, 10], increased ambulance diversion [35, 7-11], decreased regional disaster response capacity [3], increased morbidity and mortality [3, 4, 7, 9, 10, 16-18], increased number of patients returning to the ED [7], and ED staff dissatisfaction and burnout $[5,8,10]$. Crowding is attributed to $31 \%$ of sentinel events in the ED [4].

In the United States, the average ED LOS, defined as the elapsed time from patient registration until the patient physically departs, increased from 132 minutes in 2001 to 154 minutes in 2005. These numbers have drastically increased in the subsequent decade, as is witnessed in the literature and in our own experience [1-5]. Although LOS is not a direct measure of crowding, it is an easily quantifiable surrogate marker for ED crowding. Moreover, LOS is a useful measure associated with ED quality, timeliness, patient-centeredness, safety, patient satisfaction, and efficiency in emergency care $[3,8,9,13]$.

The literature shows that triage orders have been shown to decrease LOS, decrease time to time-sensitive interventions, increase patient comfort, improve throughput, and improve employee satisfaction [5]. In this study, we evaluated standing triage orders as a strategy to improve ED throughput; we looked specifically at the effect of standing orders on ED LOS and the time to disposition after provider evaluation.

For patients presenting with chest pain, if all labs resulted prior to provider evaluation (63\% of patients), disposition time decreased by 26 minutes $(16.9 \%, p=0.04)$. These decreases were found to be statistically significant. Moreover, a $16.9 \%$ decrease in time to disposition is clinically significant, especially in a busy, overcrowded emergency department setting where standing orders are being implemented to facilitate throughput.

Retezar et al. [8] performed a similar retrospective study evaluating the effect of triage standing orders on ED treatment time. They found that the median ED treatment time decreased from 282 minutes for patients who did not receive triage orders to 230 minutes (18\% decrease) for patients who received at least partial triage orders [8].

We initially hypothesized that standing orders would decrease ED LOS. However, our results demonstrate that the implementation of standing orders was associated with prolonged ED LOS. For patients presenting with chest pain, ED LOS increased from 402 min (without standing orders) to 614 min (with standing orders). This can be explained due to the nature of standing orders being used during busier throughput times. During high census periods in the ED and hospital, standing orders are implemented when patients are unable to be roomed or seen by a provider in a timely fashion. Even though the standing orders may be completed, patients continue to sit in the waiting room and cannot be evaluated because there are no rooms for patients to be evaluated 
TABLE 2: Standing orders decrease disposition time for patients presenting with chest pain. Moreover, when all tests result prior to provider evaluation, disposition time decreases even further. ED LOS, however, increases with the implementation of standing orders, reflecting the use of standing orders during high census periods in the ED.

\begin{tabular}{|c|c|c|c|c|c|}
\hline & \multicolumn{2}{|c|}{ Standing orders $(n=149)$} & \multicolumn{2}{|c|}{ No standing orders $(n=152)$} & \multirow{2}{*}{$p$ value } \\
\hline & Median (min) & Quartiles (min) & Median (min) & Quartiles (min) & \\
\hline Total ED LOS (min) & 614 & $461-841$ & 402 & 303-639 & $<0.001$ \\
\hline Provider to disposition (min) & 128 & $73-274$ & 154 & $106-258$ & 0.042 \\
\hline
\end{tabular}

in, prolonging their LOS. Once a provider evaluates the patients, their disposition time is shortened because results are available for medical decision-making. Thus, crowding affects wait times and ED LOS but not treatment time, as is seen in several other studies $[8,13,19]$.

Standing triage orders initiate the diagnostic evaluation earlier, although its scope is limited to the order set itself. Additional clinical factors, including vital signs, history of present illness, past medical and family history, and physical exam, may dictate further workup and testing (i.e., d-dimer, computed tomography to evaluate for aortic dissection or pulmonary embolism, and ABG). These elements can only be elicited by a provider and cannot be reflexively included in a standing order set. An average of 3.7 additional labs and 1.3 additional imaging studies were ordered after provider evaluation of patients presenting with chest pain.

Retezar et al. [8] showed that the majority of patients (98\%) who did not receive standing orders received similar workups after being evaluated by a provider. Therefore, the implementation of standing orders initiates a similar workup in patients who cannot be evaluated immediately. It may be argued that since the orders are similar between the standing orders and no-standing orders cohort, the timeline is essentially shifted to begin earlier in the standing orders cohort (prior to provider evaluation) instead of after provider evaluation. However, had standing orders not been implemented, patients would have to wait until being roomed and evaluated by a provider before any labs were ordered or have resulted, significantly prolonging both disposition and ED LOS. Moreover, the initiation of the standing orders decreases time-to-time sensitive interventions (i.e., aspirin for cardiac chest pain and cardiac catheterization lab activation for STEMI) which improves quality and patient care.

The causes of overcrowding can be broadly categorized into input, throughput, and output factors. However, the causes are interrelated and multifaceted and include increased medical complexity and acuity, increased overall patient volume, a lack of inpatient beds, shortages of physicians, nurses, and staff, delays in laboratory or radiology results, and limited space within the ED [20-23]. Besides the implementation of standing orders, numerous studies demonstrate that other changes in throughput, such as modifications to capacity, triage, laboratory testing, staffing, and registration, improve ED LOS [7, 8, 24-27]. Along the same lines, the placement of a physician or midlevel provider at triage is an alternative strategy to triage standing orders [5, 28]. Previous studies evaluating the placement of a physician in triage have demonstrated mixed results with significant limitations $[5,11,29]$. However, empowering triage nurses to order analgesia, blood tests, and radiography reduces the median time for pain assessment ( $47 \mathrm{~min}$ to $1 \mathrm{~min}$ ), the median time for analgesia administration (98 $\mathrm{min}$ to $28 \mathrm{~min}$ ), waiting times, and ED LOS, while improving patient satisfaction, quality of care, and throughput [30-34].

However, it has been suggested that the largest contributor to ED crowding is due to "throughput" and "output" factors rather than unchangeable "input" factors $[4,9,13]$. Herring et al. found that ED LOS increased by 22 minutes (17\%) between 2001 and 2005 [3]. Moreover, the trend in prolonged LOS disproportionately affects critically ill patients admitted to intensive care units, with an increase more than twice as large (69 minutes, 37\%), because the lack of inpatient beds and resources disproportionately affect critically ill patients. As the current population ages with more comorbidities, these increases will become even more pronounced for patients with acute illnesses $[3,9,10]$. Current research strongly suggests that ED crowding is affected more by output factors, such as hospital occupancy, availability of inpatient beds, and the ability to transfer patients to inpatient beds $[4,9,13]$.

The results of this study must be interpreted in the context of the following limitations. First, this study was a retrospective cohort analysis; the patients were not randomized to either the standing order or no-standing order cohort. After the implementation of standing orders in April 2014, patients presumably did not receive standing orders if the emergency department was less crowded and patients could be seen reasonably quickly. Therefore, to eliminate bias and Hawthorne effect, the no-standing order cohort was selected from patients seen prior to the implementation of standing orders, while the standing order cohort was selected from patients seen after standing orders were implemented. The two groups were similar in age, gender, and illness severity, although other differences may not be readily apparent (e.g., comorbidities and previous surgeries) and may potentially confound our findings.

Second, this study evaluated the effect of standing orders on patients presenting with chest pain. Future studies will be needed to evaluate whether standing orders impact other patient complaints similarly. Third, we did not include information on which additional lab and imaging tests were ordered after provider evaluation. If providers consistently ordered certain tests, it may be worthwhile to consider adding these tests as part of the order set to further expedite care.

Fourth, the generalizability of this single center large academic ED study is limited; our hospital ED may not be a representative sample of hospital EDs throughout the United States. Other facilities may work up certain complaints with 
different lab and imaging studies. The standing triage order set was developed according to the general practice and consensus of attending physicians at our facility and may not be applicable elsewhere. However, we believe that the implementation of standing orders based on a local consensus will have similar results.

Finally, how standing orders affect long-term morbidity and mortality would be important quality measures that were not studied. A thorough multiinstitutional prospective study assessing the effect of triage orders on quality outcomes, ED costs, and throughput is needed.

\section{Conclusion}

In conclusion, standing orders are implemented when there is a bottleneck within the ED and patients are unable to be evaluated expeditiously. Although this bottleneck results in a prolonged LOS, this study demonstrated that the implementation of standing orders expedites disposition time. Moreover, standing orders initiate the diagnostic workup and allow the patient access to care, earlier during the patient's ED stay which is especially important in time-sensitive conditions. Appropriately designed standing orders are similar to provider orders and few additional orders are needed. What is not clear from this study is which additional studies should also be implemented in the standing order set. In addition, future adequately powered randomized controlled studies need to be performed for various chief complaints.

ED crowding is a local manifestation of a system-wide problem that incorporates input, throughput, and output factors, many of which are not under the control of the ED but instead are influenced by hospital- and communitylevel factors. Standing orders reduce ED disposition times but are being implemented when overall ED crowding and throughput are compromised and therefore cannot show an impact on overall ED LOS.

\section{Disclosure}

The authors alone are responsible for the content and writing of the paper.

\section{Competing Interests}

The authors report no declarations of interest.

\section{References}

[1] E. W. Nawar, R. W. Niska, and J. Xu, "National Hospital Ambulatory Medical Care Survey: 2005 emergency department summary," Advance Data, no. 386, pp. 1-32, 2007.

[2] Institute of Medicine. Committee on the Future of Emergency Care in the United States Health System, Hospital-Based Emergency Care: At the Breaking Point, National Academy Press, Washington, DC, USA, 2006.

[3] A. Herring, A. Wilper, D. U. Himmelstein et al., "Increasing length of stay among adult visits to U.S. Emergency departments, 2001-2005," Academic Emergency Medicine, vol. 16, no. 7, pp. 609-616, 2009.
[4] J. C. Moskop, D. P. Sklar, J. M. Geiderman, R. M. Schears, and K. J. Bookman, "Emergency department crowding, part 2barriers to reform and strategies to overcome them," Annals of Emergency Medicine, vol. 53, no. 5, pp. 612-617, 2009.

[5] J. L. Wiler, C. Gentle, J. M. Halfpenny et al., "Optimizing emergency department front-end operations," Annals of Emergency Medicine, vol. 55, no. 2, pp. 142-160.el, 2010.

[6] S. A. Hahn, E. J. Lavonas, S. E. Mace et al., "Clinical policy: critical issues in the initial evaluation and management of patients presenting to the emergency department in early pregnancy," Annals of Emergency Medicine, vol. 41, no. 1, pp. 123133, 2003.

[7] S. L. Bernstein, D. Aronsky, R. Duseja et al., "The effect of emergency department crowding on clinically oriented outcomes," Academic Emergency Medicine, vol. 16, no. 1, pp. 1-10, 2009.

[8] R. Retezar, E. Bessman, R. Ding, S. L. Zeger, and M. L. McCarthy, "The effect of triage diagnostic standing orders on emergency department treatment time," Annals of Emergency Medicine, vol. 57, no. 2, pp. 89-99.e2, 2011.

[9] L. I. Horwitz, J. Green, and E. H. Bradley, "US emergency department performance on wait time and length of visit," Annals of Emergency Medicine, vol. 55, no. 2, pp. 133-141, 2010.

[10] N. R. Hoot and D. Aronsky, "Systematic review of emergency department crowding: causes, effects, and solutions," Annals of Emergency Medicine, vol. 52, no. 2, pp. 126-136.e1, 2008.

[11] J. H. Han, D. J. France, S. R. Levin, I. D. Jones, A. B. Storrow, and D. Aronsky, "The effect of physician triage on emergency department length of stay," Journal of Emergency Medicine, vol. 39, no. 2, pp. 227-233, 2010.

[12] M. M. Dinh, N. Enright, A. Walker, A. Parameswaran, and M. Chu, "Determinants of patient satisfaction in an Australian emergency department fast-track setting," Emergency Medicine Journal, vol. 30, no. 10, pp. 824-827, 2013.

[13] M. L. McCarthy, S. L. Zeger, R. Ding et al., "Crowding delays treatment and lengthens emergency department length of stay, even among high-acuity patients," Annals of Emergency Medicine, vol. 54, no. 4, pp. 492-503.e4, 2009.

[14] K. J. Hong, S. D. Shin, K. J. Song, W. C. Cha, and J. S. Cho, "Association between ED crowding and delay in resuscitation effort," American Journal of Emergency Medicine, vol. 31, no. 3, pp. 509-515, 2013.

[15] R. Sikka, S. Mehta, C. Kaucky, and E. B. Kulstad, "ED crowding is associated with an increased time to pneumonia treatment," American Journal of Emergency Medicine, vol. 28, no. 7, pp. 809$812,2010$.

[16] D. B. Richardson, "Increase in patient mortality at 10 days associated with emergency department overcrowding," Medical Journal of Australia, vol. 184, no. 5, pp. 213-216, 2006.

[17] P. C. Sprivulis, J.-A. Da Silva, I. G. Jacobs, A. R. L. Frazer, and G. A. Jelinek, "The association between hospital overcrowding and mortality among patients admitted via Western Australian emergency departments," Medical Journal of Australia, vol. 184, no. 5, pp. 208-212, 2006.

[18] P. A. Cameron, "Hospital overcrowding: a threat to patient safety?" Medical Journal of Australia, vol. 184, no. 5, pp. 203204, 2006.

[19] P. V. Asaro, L. M. Lewis, and S. B. Boxerman, "The impact of input and output factors on emergency department throughput," Academic Emergency Medicine, vol. 14, no. 3, pp. 235-242, 2007. 
[20] K. Crawford, J. Morphet, T. Jones, K. Innes, D. Griffiths, and A. Williams, "Initiatives to reduce overcrowding and access block in Australian emergency departments: a literature review," Collegian, vol. 21, no. 4, pp. 359-366, 2014.

[21] N. Jayaprakash, R. O'Sullivan, T. Bey, S. S. Ahmed, and S. Lotfipour, "Crowding and delivery of healthcare in emergency departments: the European perspective," The Western Journal of Emergency Medicine, vol. 10, no. 4, pp. 233-239, 2009.

[22] R. W. Derlet and J. R. Richards, "Overcrowding in the nation's emergency departments: complex causes and disturbing effects," Annals of Emergency Medicine, vol. 35, no. 1, pp. 63$68,2000$.

[23] Ò. Micró, M. Sánchez, G. Espinosa, B. Coll-Vinent, E. Bragulat, and J. Millá, "Analysis of patient flow in the emergency department and the effect of an extensive reorganisation," Emergency Medicine Journal, vol. 20, no. 2, pp. 143-148, 2003.

[24] C. M. B. Fernandes, J. M. Christenson, and A. Price, "Continuous quality improvement reduces length of stay for fast-track patients in an emergency department," Academic Emergency Medicine, vol. 3, no. 3, pp. 258-263, 1996.

[25] M. H. Gorelick, K. Yen, and H. J. Yun, "The effect of in-room registration on emergency department length of stay," Annals of Emergency Medicine, vol. 45, no. 2, pp. 128-133, 2005.

[26] J. M. Hirshon, T. D. Kirsch, W. K. Mysko, and G. D. Kelen, "Effect of rotational patient assignment on emergency department length of stay," Journal of Emergency Medicine, vol. 14, no. 6, pp. 763-768, 1996.

[27] A. J. Singer, J. Ardise, J. Gulla, and J. Cangro, "Point-of-care testing reduces length of stay in emergency department chest pain patients," Annals of Emergency Medicine, vol. 45, no. 6, pp. 587-591, 2005.

[28] F. Subash, F. Dunn, B. McNicholl, and J. Marlow, "Team triage improves emergency department efficiency," Emergency Medicine Journal, vol. 21, no. 5, pp. 542-544, 2004.

[29] B. R. Holroyd, M. J. Bullard, K. Latoszek et al., "Impact of a triage liaison physician on emergency department overcrowding and throughput: a randomized controlled trial," Academic Emergency Medicine, vol. 14, no. 8, pp. 702-708, 2007.

[30] J. C. Finn, A. Rae, N. Gibson, R. Swift, T. Watters, and I. G. Jacobs, "Reducing time to analgesia in the emergency department using a nurse-initiated pain protocol: a before-andafter study," Contemporary Nurse, vol. 43, no. 1, pp. 29-37, 2012.

[31] J. Tambimuttu, R. Hawley, and A. Marshall, "Nurse-initiated $\mathrm{X}$-ray of isolated limb fractures in the emergency department: research outcomes and future directions," Australian Critical Care, vol. 15, no. 3, pp. 119-122, 2002.

[32] J. Streppa, V. Schneidman, and A. D. Biron, "Requesting wrist radiographs in emergency department triage: developing a training program and diagnostic algorithm," Advanced Emergency Nursing Journal, vol. 36, no. 1, pp. 62-77, 2014.

[33] J. Considine, E. Lucas, R. Payne, M. Kropman, H. E. Stergiou, and $\mathrm{H}$. Chiu, "Analysis of three advanced practice roles in emergency nursing," Australasian Emergency Nursing Journal, vol. 15, no. 4, pp. 219-228, 2012.

[34] K. Innes, D. Jackson, V. Plummer, and D. Elliott, "Care of patients in emergency department waiting rooms-an integrative review," Journal of Advanced Nursing, vol. 71, no. 12, pp. 2702-2714, 2015. 


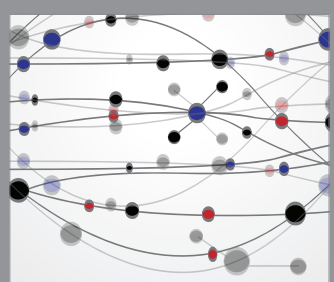

The Scientific World Journal
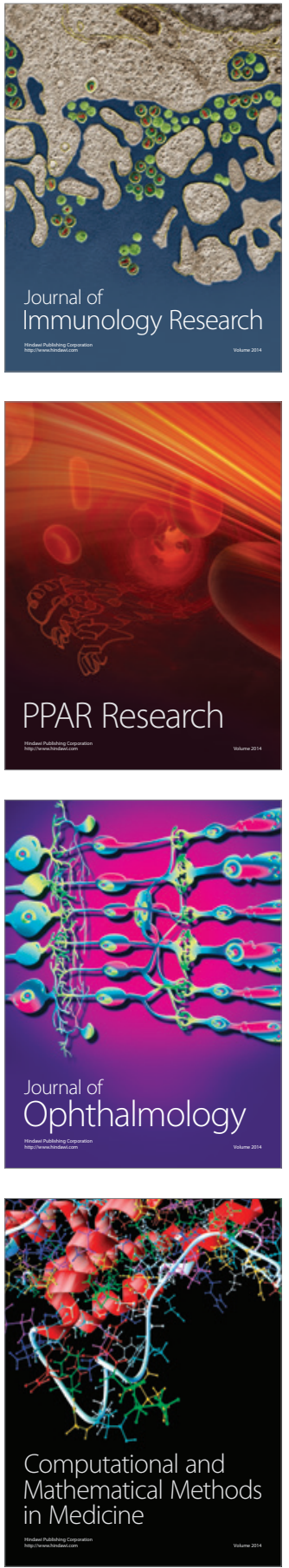

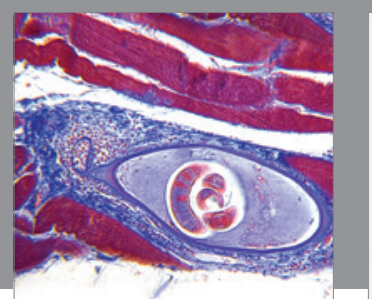

Gastroenterology Research and Practice

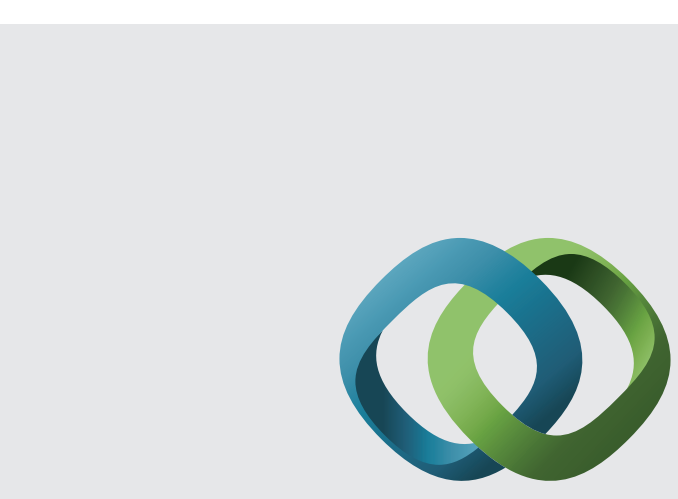

\section{Hindawi}

Submit your manuscripts at

http://www.hindawi.com
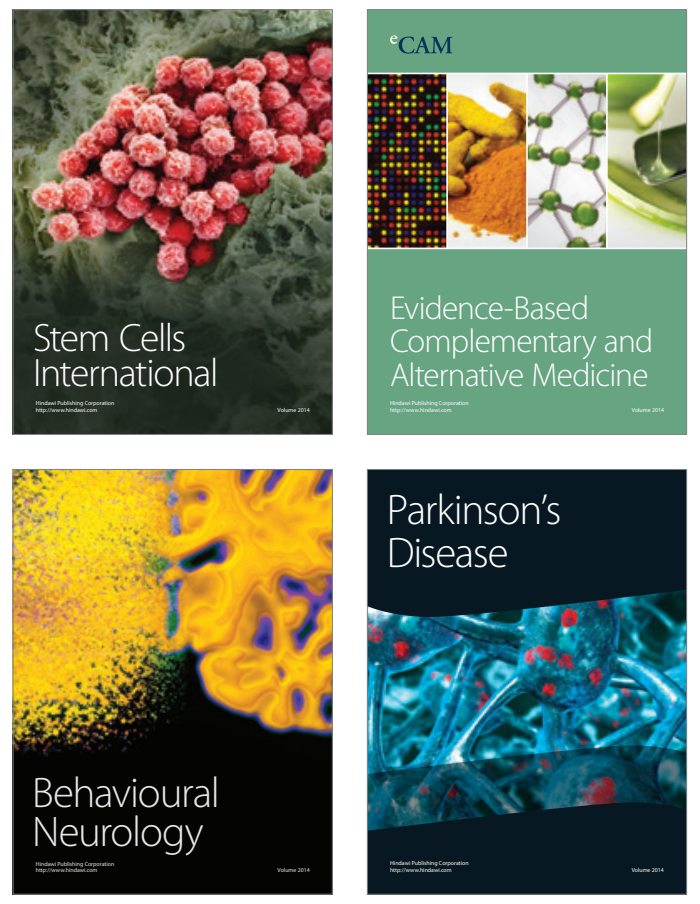
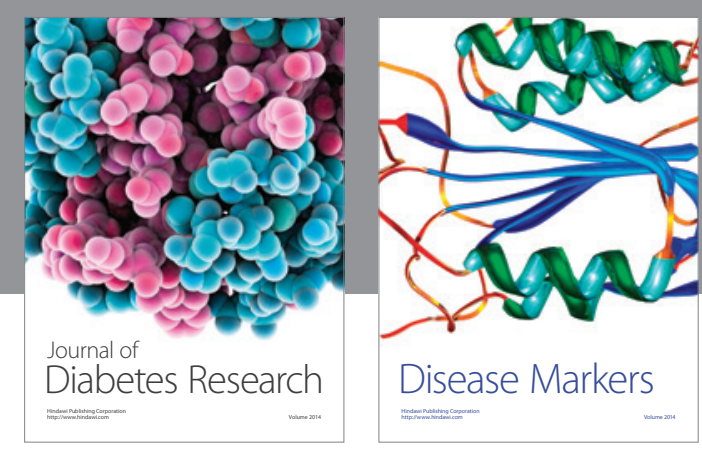

Disease Markers
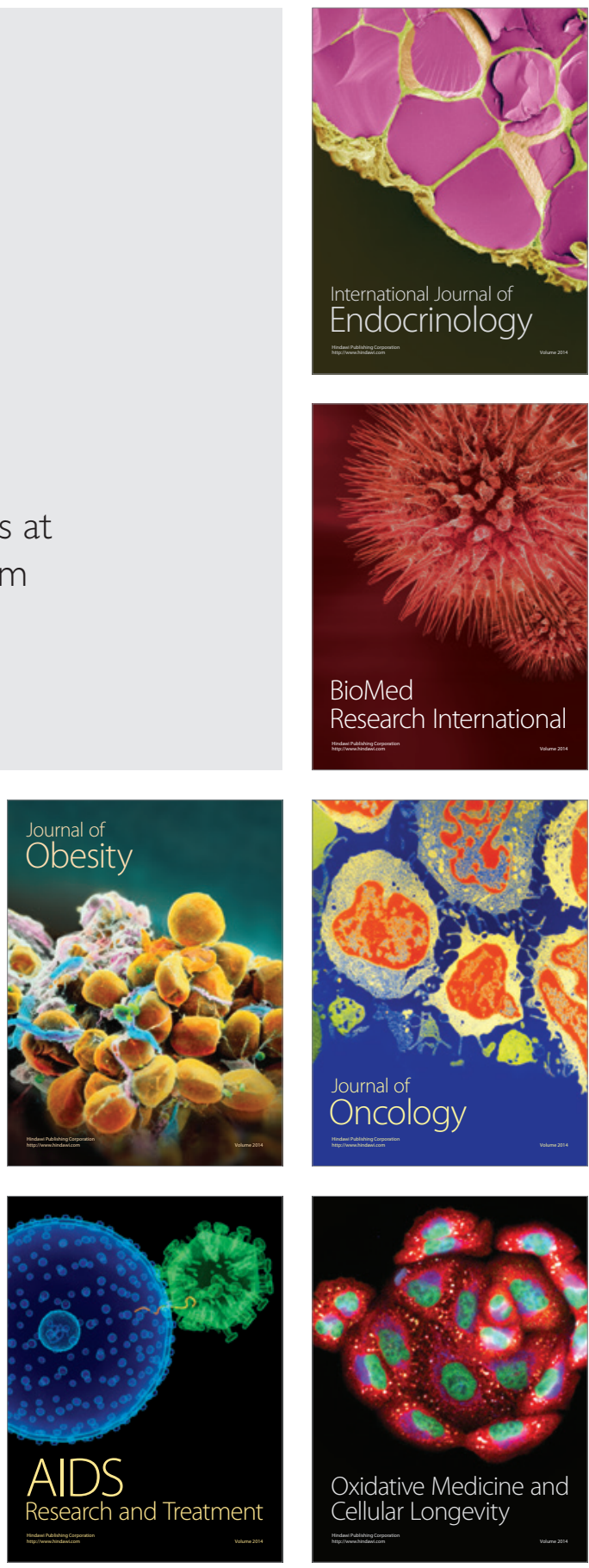